\title{
Prenatal nutritional high fat environment and the evolving concept of intergenerational cardio- metabolic disease risk
}

Keywords: Blood pressure, cardiovascular dysfunction, developmental programming, HMG-CoA reductase inhibition, endothelial progenitor cells

\section{Editorial}

The most rapid and dramatic consequence in the course of human history with the advent of the global economy are the changes in eating patterns, though undoubtedly fuelled by urbanization. Radical dietary shifts in many developed and developing nations are supplanting traditional patterns of eating with Western diet high in animal products, refined carbohydrates and low in whole grains, fruits, and vegetables. For example, in China, consumption of animal products increased by nearly $40 \%$ between 1989 and $1997^{1}$ and high fat HF food sales more than doubled between 1999 and 2005. ${ }^{1}$ The term "Coca-colonization," a reference to the ubiquitous presence of Coca-Cola, Pepsi, and McDonald's, describes a world that is moving toward a common diet, one accompanied by the more sedentary lifestyles associated with increased risk of chronic disease. ${ }^{2,3}$

According to the World Health Organization's global database, countries like China, India and Pakistan have a preschool childhood obesity prevalence of about $1 \% \cdot{ }^{4}$ Childhood obesity increases the risk of obesity in adulthood and parental obesity interacts quite strongly to alter this risk. There are several other interactive factors as well contributing to the increased prevalence of obesity in childhood. Societies like China, India and Pakistan, which are rapidly urbanising, demonstrate increases in energy intake, dramatic increases in fat intake along with increased levels of sedentarianism. Food balance data from the Food and Agriculture Organization show that the change in energy intake in Asian countries has been small, but there have been large changes in consumption of animal products, sugars and fats. ${ }^{5}$ The net effect has been a marked shift in the diet with energy from fat (both animal and vegetable) increasing each year. ${ }^{5}$ Data from India show that higher-income groups consumed a diet with $32 \%$ of the energy from fat while the lower-income groups consumed only $17 \%$ energy from fat. ${ }^{6-9}$

Therefore, nutritional history of current populations becomes an important determinant of their health. There is a growing recognition of the importance of environmental factors acting on the genotype throughout the life cycle of an individual to progressively modify its phenotype. The periconceptional and intrauterine period seem to be the most crucial, when a small change in environment could have a large effect on the phenotype. The slogan of the UN Expert Meeting held in April 2008, "Woman's Health is a Nation's Wealth" reflects this philosophy. ${ }^{10}$ We have demonstrated that maternal high fat diet causes hypercholesterolemic mediated teratogenesis with deranged lipid profile, increased oxidative stress and decreased activity of endothelial progenitor cells in the circulation of the offspring. ${ }^{11-13}$ Our hypothesis of high fat diet mediates hypercholesterolemia in young female mammals may indicate a similar phenomenon in humans, a possible significant factor in the escalating epidemic of cardio-
Volume 3 Issue 2 - 2016

\author{
Maqsood M Elahi,' Bashir M Matata ${ }^{2}$ \\ 'Heart-Lung Research Institute, Pakistan \\ ${ }^{2}$ Department of Clinical Research, United Kingdom
}

Correspondence: Bashir Matata, Department of Clinical Research, The Liverpool Heart \& Chest Hospital NHS Foundation Trust, Liverpool, United Kingdom,

Email matata_bashir@hotmail.com

Received: June 01, 2016 | Published: June 02, 2016

metabolic syndrome. In a female, such a situation exposes her foetus to multiple adverse programming influences, resulting in a complex phenotype including exaggerated tendency to develop adiposity, obesity, hypertension, hypercholesterolemia and hyperinsulinemia at a young age. ${ }^{12}$

Hence in our hypothesis "Hypercholesterolemic-mediated Teratogenesis Model ${ }^{14}$ " we suggested that over-nutritional high fat environment prenatally and postnatally may shift the balance between postnatally healthy survival and survival but with increased disease risk. For example the potentiation of increased CVD risk with early manifestation of cardio-metabolic syndrome for the offspring during adult life. From an adequate nutritional environment, the offspring then lies outside the band of rich and very rich environment that can be detrimental to its survival fitness. Hypothetically, administration of an early pharmacological treatment (such as statins during the late stages of trimester) to mothers on high fat diet, even at rich nutritional upper limit, could provide a good safeguard from the disease process and maintain the survival fitness even if increasing affluence raises the richness of the mature postnatal environment. ${ }^{11,13,14}$ In humans, the reality is that most pharmacological interventions are not practical since such agents are likely to be contra-indicated for pregnant mothers e.g. statins etc.

The basic principle of prevention of cardio-metabolic syndrome events consists of the control of classical risk factors with specific interventions. This clinical 'high-risk' approach has led to an improvement in prognosis, although still far below expectations. In other words, the mere control of hypertension, hypercholesterolemia, or obesity, though definitely beneficial, leaves a residual risk, which is still greater than that reduced by treatment. Thus, it is necessary to identify windows of time in the lifecycle when the genome is highly susceptible to such influences. Such knowledge could lead to the discovery of different treatment targets that may have a bigger impact upon CVD burden. Any preventive intervention will therefore have to start in utero, and improving the health of young girls will be a very important aspect of such an approach. 
The cardio-metabolic programming effect of maternal hypercholesterolemia and the observation of strikingly high foetal cholesterol levels at the end of $2^{\text {nd }}$ trimester raise interest in maternalfetal cholesterol transport. It is speculated that maternal cholesterol might cross the placental barrier such as in Smith Lemli Opitz syndrome where survival of foetus depends on maternal cholesterol. ${ }^{15}$ Identification of active transport mechanisms on the maternal side of the placenta in rabbits ${ }^{16}$ and hamsters ${ }^{17}$ has provided evidence for maternal-foetal cholesterol transport. The trans-placental cholesterol transport mechanism mediated by endothelial cells lining the placental villi into the lumen of foetal microvessels has recently been elucidated. ${ }^{18}$ Results indicate that regulation of these mechanisms by increased foetal demand for cholesterol differs from that induced by maternal hypercholesterolemia. ${ }^{19}$ Increased cholesterol transport, at least during parts of gestation may therefore constitute one mechanism by which maternal hypercholesterolemia affects foetal programming and hence requires further investigations.

Yet, epigenetic changes associated with increased disease susceptibility such as obesity, type- 2 diabetes and cardiovascular dysfunction are emerging as critical components of enduring effects induced by early life experiences. The available data are now beginning to provide a molecular basis for involvement of oxidative stress in modifying biological properties of DNA methylation and other epigenetic effects. ${ }^{20}$ It is reported that in utero conditions result in specific epigenetic change. ${ }^{21,22}$ Developing organisms seem to have a wide window of susceptibility to epigenetic changes but the periconceptional period is particularly important towards sensitivity to suboptimal nutrition during this developmental stage in which widespread reprogramming of the epigenome occurs. ${ }^{23-25}$ It is speculated that HF over-nutrition in pregnancy and/or postnatal could also lead to metabolic dysfunction in major organs such as kidneys and liver later on in life. ${ }^{26-30}$ These epigenetic changes within suboptimal nutrition could have long-lasting effects on epigenomic methylation patterns in specific gene promoters. Moreover, the study of epigenetics will further guide us in understanding the hormonal regulation of enzymes controlling acetylation and methylation and sex differences across the epigenome.

Therefore, from all the discussion, it will be ideal to demonstrate (1) adverse effects of impaired early-life maternal HF nutrition associated epigenetic changes in offspring and (2) the mechanisms/impact of early interventions such as statins in mothers on HF diet for prevention or reversal of such epigenetic consequences during intrauterine development. Today, we have to recognize that it may be still too early to test these hypotheses in the clinical setting, yet the animal models have established cause-effect relationships for some maternal factors and led to the identification of specific questions in a high fat maternal environments. Despite this, mechanistic studies, both in animals and in humans, are needed in order to achieve a better understanding of the role of maternal high fat diet in the pathophysiology of cardiometabolic syndrome. In addition, development of methods that would allow better characterization of this cardio-metabolic milieu in the humans is necessary. Only a more in-depth knowledge of these important pathophysiological mechanisms can allow the identification and testing of novel therapeutic strategies aimed at targeting the early developmental origins of cardiovascular disease in the populations.

So far from our own work, ${ }^{11,13}$ we have to some extent demonstrated in animal models the effects of long term maternal high fat diet and statin treatment on the offspring predisposition to early cardiometabolic metabolic disease. However, much of our understanding of the disorder is far from complete. Future research originating from present work could focus on the translation of unexplored signalling pathways to answer the central question such as, how do such maternal high fat diets affect the long term cardiovascular health of offspring.

Throughout foetal development, the main nutritional source for the foetus comes from the mother. As a result, the quality of the maternal diet affects growth and development of the foetus during this period ${ }^{31}$ though the mechanisms involved are poorly understood. Nevertheless, experimental animal models whereby the maternal diet is manipulated have provided insight into the links between in-utero environments and susceptibility to disease in later life (Figure 1). Rodent offspring from dams that were fed a protein-restricted diet during pregnancy later developed hypertension in adulthood. ${ }^{32}$ However, it is fair to suggest that developmental programming of cardio-metabolic syndrome in adulthood is not limited to maternal dietary restriction alone. Ours and other groups have shown that feeding dams a high fat diet during pregnancy and/or lactation can also leads to the offspring developing vascular dysfunction and hypertension ${ }^{11,13,33,34}$ and that changes in the maternal diet during pregnancy are linked with an increase in cardiomyocytes and foetal left ventricular mass. ${ }^{35}$

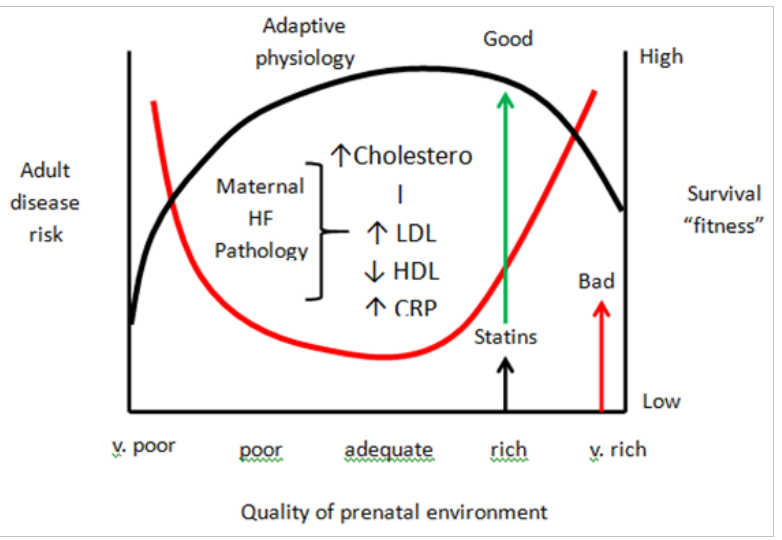

Figure I The hypothesized "Hypercholesterolemic-mediated Teratogenesis" model. This model demonstrates on one extreme (right side) the risk of adult disease and on the other extreme (left side) range of postnatal physiological settings for survival fitness. At the $x$-axis (bottom) five different qualities of prenatal environments from very poor to very rich are presented. The red line curve represents the environment that the foetus anticipates postnatally judged from the nutritional and related signals it receives from the mother through the placenta. Provided that the postnatal environment matches the range for which the foetus has set its postnatal physiology by the processes of predictive adaptive responses, the disease risk is low. However, when it lies outside that range then the postnatal environment turns bad (indicated by the red arrow) and the disease risk increases. The foetus perceives the more restricted environment and predicts a poorer postnatal survival. But maternal high-fat dietary stress worsens the situation. Over-nutritional high fat environment prenatally and postnatally shifts the postnatal survival associated with increased disease risk further to the upper limit (represented with curved black line). However, administration of statin to mothers on high fat diet, even at rich nutritional upper limit, can provide a good safeguard from the disease process and maintains the survival fitness even if increasing affluence raises the richness of the mature postnatal environment.

Cardiomyocytes go through terminal differentiation at or after birth and this is characterised by the transition from hyperplastic growth to hypertrophic growth. ${ }^{36-38}$ Many genes are reported to be involved in cell cycle progression and are associated with cardiac hypertrophy. ${ }^{39} \mathrm{It}$ remains however to be determined whether the hypercholesterolemic condition during pregnancy leads to any long term changes in these 
genes in the adult offspring's heart; and whether the cholesterollowering effect of statin given to pregnant dams consuming a high fat diet influence the expression of these genes.

\section{Conclusion}

Recent studies have provided insights into the relationship between maternal nutritional status and intergenerational risk of cardio-metabolic disease. Evidence derived from animal models has concluded that high fat diet coupled with sedentary lifestyles provokes a cardio-metabolic trait whose effect transcends at least two offspring generations. However, such knowledge is still considered hypothetical in the absence of randomised clinical trials in the human. Development of interventions is limited by the absence of research at molecular/cell levels to identify potential genetic targets and at the human levels where many questions still remain answered.

\section{Acknowledgements}

None.

\section{Conflict of interest}

The author declares no conflict of interest.

\section{References}

1. Popkin BM, Du S. Dynamics of the nutrition transition toward the anima foods sector in China and its implications: a worried perspective. J Nutr. 2003;133(11 Suppl 2):3898-3906.

2. Frazão E, Meade B, Regmi A. Converging patterns in global food consumption and food delivery systems. Amber Waves. 2008;6:22-29.

3. Zimmet P. Globalization, coca-colonization and the chronic disease epidemic: can the doomsday scenario be averted? J Intern Med 2000;247(3):301-310.

4. World Health Organization. Obesity: preventing and managing the global epidemic. report of a WHO consultation. World Health Organ Tech Rep Ser. 2000;894:i-xii, 1-253.

5. Food and agriculture organization (FAO)/World health organization. Fats and oils in human nutrition. Report of a joint expert consultation. FAO Food Nutr Pap. 1994;57:i-xix, 1-147.

6. Dhurandhar NV, Kulkarni PR. Prevalence of obesity in Bomaby. Int J Obes Relat Metab Disord. 1992;16(5):367-375.

7. Gopinath N, Chadha SL, Jain P, et al. An epidemiological study of obesity in adults in the urban population of Delhi. J Assoc Physicians India. 1994;42(3):212-215.

8. Zargar AM, Masoodi SR, Laway BA, et al. Prevalence of obesity in adults - an epidemiological study from Kashmir valley of Indian subcontinent. $J$ Assoc Physicians India. 2000;48(12):1170-1174.

9. Gopalan C. Obesity in the urban middle class. NFI Bull. 1998;19:1-4.

10. World diabetes foundation, Global alliance for women's health. Diabetes, women, and development. Meeting summary, expert recommendations for policy action, conclusions, and follow-up actions. Int J Gynecol Obstet. 2009;104 Suppl 1:S46-50.

11. Elahi MM, Cagampang FR, Anthony FW, et al. Statin treatment in hypercholesterolemic pregnant mice reduces cardiovascular risk factors in their offspring. Hypertension. 2008;51(4):939-944.

12. Elahi MM, Cagampang FR, Mukhtar D, et al. Long-term maternal highfat feeding from weaning through pregnancy and lactation predisposes offspring to hypertension, raised plasma lipids and fatty liver in mice. $\mathrm{Br} J$ Nutr. 2009;102(4):514-519.

13. Elahi MM, Cagampang FR, Ohri SK, et al. Long-term statin administration to dams on high-fat diet protects not only them but also their offspring from cardiovascular risk. Ann Nutr Metab. 2013;62(3):250-256.

14. Maqsood M Elahi, Bashir M Matata. Hypercholesterolemic-mediated Teratogenesis: Materno-Fetal Cholesterol Hypothesis of Cardiovascular Disease” LAP LAMBERT Academic Publishing GmbH \& Co KG; 2012.

15. Lindegaard ML, Wassif CA, Vaisman B, et al. Characterization of placental cholesterol transport: ABCA1 is a potential target for in utero therapy of Smith-Lemli-Opitz syndrome. Hum Mol Genet. 2008;17(23):3806-3813.

16. Montoudis A, Simoneau L, Brissette L, et al. Impact of a cholesterol enriched diet on maternal and fetal plasma lipids and fetal deposition in pregnant rabbits. Life Sci. 1999;64(26):2439-2450.

17. McConihay JA, Horn PS, Woollett LA. Effect of maternal hypercholesterolemia on fetal sterol metabolism in the Golden Syrian hamster. J Lipid Res. 2001;42(7):1111-1119.

18. Stefulj J, Panzenboeck U, Becker T, et al. Human endothelial cells of the placental barrier efficiently deliver cholesterol to the fetal circulation via ABCA1 and ABCG1. Circ Res. 2009;104(5):600-608.

19. Palinski W. Maternal-fetal cholesterol transport in the placenta: good, bad, and target for modulation. Circ Res. 2009;104(5):569-571.

20. Gluckman PD, Hanson MA, Cooper C, et al. Effect of in utero and earlylife conditions on adult health and disease. N Engl J Med. 2008;359(1):6173.

21. Tarry-Adkins JL, Chen JH, Smith NS, et al. Poor maternal nutrition followed by accelerated postnatal growth leads to telomere shortening and increased markers of cell senescence in rat islets. FASEB J. 2009;23(5):1521-1528.

22. Heijmans BT, Tobi EW, Stein AD, et al. Persistent epigenetic differences associated with prenatal exposure to famine in humans. Proc Natl Acad Sci US A. 2008;105(44):17046-17049.

23. Gluckman PD, Hanson MA. Living with the past: evolution, development, and patterns of disease. Science. 2004;305(5691):1733-1736.

24. Gluckman PD, Hanson MA, Pinal C. The developmental origins of adult disease. Matern Child Nutr. 2005;1(3):130-141.

25. Gluckman PD, Hanson MA, Buklijas T, et al. Epigenetic mechanisms that underpin metabolic and cardiovascular diseases. Nat Rev Endocrinol. 2009;5(7):401-408.

26. Brawley L, Dance CS, Dunn RL, et al. Dietary folate supplementation prevents the attenuated relaxation to vascular endothelial growth factor (VEGF) in the uterine artery of protein-restricted pregnant rats. Pediatr Res. 2003;53:37A.

27. Brawley L, Torrens C, Anthony FW, et al. Glycine rectifies vascular dysfunction induced by dietary protein imbalance during pregnancy. $J$ Physiol. 2004;554(Pt 2):497-504.

28. Lillycrop KA, Phillips ES, Jackson AA, et al. Dietary protein restriction of pregnant rats induces and folic acid supplementation prevents epigenetic modification of hepatic gene expression in the offspring. J Nutr. 2005;135(6):1382-1386.

29. Vickers MH, Gluckman PD, Coveny AH, et al. Neonatal leptin treatment reverses developmental programming. Endocrinology. 2005;146(10):4211-4216.

30. Gluckman PD, Lillycrop KA, Vickers MH, et al. Metabolic plasticity during mammalian development is directionally dependent on early nutritional status. Proc Natl Acad Sci USA. 2007;104(31):12796-12800. 
31. Roseboom TJ, van der Meulen JH, Osmond C, et al. Coronary heart disease after prenatal exposure to the Dutch famine, 1944-45. Heart 2000;84(6):595-598.

32. Langley-Evans SC. Intrauterine programming of hypertension in the rat: nutrient interactions. Comp Biochem Physiol A Physiol. 1996;114(4):327333

33. Khan I, Dekou V, Hanson M, et al. Predictive adaptive responses to maternal high-fat diet prevent endothelial dysfunction but not hypertension in adult rat offspring. Circulation. 2004;110(9):1097-1102.

34. Khan IY, Taylor PD, Dekou V, et al. Gender-linked hypertension in offspring of lard-fed pregnant rats. Hypertension. 2003;41(1):168-175.

35. Hans HC, Austin KJ, Nathanielsz PW, et al. Maternal nutrient restriction alters gene expression in the ovine fetal heart. J Physiol. 2004;558(Pt 1):111-121.
36. Claycomb WC. Cardiac- muscle hypertrophy. Differentiation and growth of the heart cell during development. Biochem J. 1977;168(3):599-601.

37. Soonpaa MH, Kim KK, Pajak L, et al. Cardiomyocyte DNA synthesis and binucleation during murine development. AMJ Physiol. 1996;271(5 Pt 2):H2183-2189.

38. Li F, Wang X, Capasso JM, et al. Rapid transition of cardiac cyocytes from hyperplasia to hypertrophy during postnatal development. J Mol Cell Cardiol. 1996;28(8):1737-1746.

39. Li JM, Brooks G. Cell cycle regulatory molecules (cyclins, cyclindependent kinases and cyclin- dependent kinase inhibitors) and the cardiovascular system; potential targets for therapy? Eur Heart J. 1999;20(6):406-420. 